\title{
Spatial structure of quark Cooper pairs in a color superconductor
}

\author{
Masayuki Matsuzaki * \\ Department of Physics, Fukuoka University of Education, Munakata, Fukuoka 811-4192, Japan
}

(July 26, 2021)

\begin{abstract}
Spatial structure of Cooper pairs with quantum numbers color $3^{*}, I=J=$ $L=S=0$ in $u d 2$ flavor quark matter is studied by solving the gap equation and calculating the coherence length in full momentum range without the weak coupling approximation. Although the gap at the Fermi surface and the coherence length depend on density weakly, the shape of the $r$-space pair wave function varies strongly with density. This result indicates that quark Cooper pairs become more bosonic at higher densities.
\end{abstract}

*E-mail address: matsuza@fukuoka-edu.ac.jp 
Color superconducting phases of strongly interacting matter at high density and low temperature [1,2,3] are attracting much attention recently. They were studied first as an example of pair condensation in relativistic many-body systems by Bailin and Love in the early 80's [4] (see also Ref. [5]). They also mentioned the nucleon-nucleon pairing as another example; its detailed study was begun in the early 90's [6] and is developing recently [7,8,9,10]. A study of the quark-quark pairing in an $\mathrm{SU}(2)$ color model was done at almost the same time [11] (see also Refs. [12,[13]). Iwasaki and Iwado's work on a 1 flavor system [14] was the first study of the realistic SU(3) color system (see also Ref. [15]). Since the works of Rapp et al. [16] and Alford et al. [17] on ud 2 flavor system, color superconductivity has been studied extensively. The ${ }^{1} S_{0}(J=L=S=0)$ state in the 2 flavor case [18] and the colorflavor-locked state in $u d s 3$ flavor case [19,20,21,22,23], respectively, have been understood to be the most favored channels. The magnetic interaction has been shown to be responsible for these pair condensations [24, 25, 26, 27,28,229]. Their astrophysical consequences were also studied [30.31].

The purpose of this Brief Report is to visualize the spatial structure, in particular its density dependence, of quark Cooper pairs, which has not been discussed, to the author's knowledge. This is done for Cooper pairs of the simplest form; color $3^{*}$, isosinglet ${ }^{1} S_{0}$ pairs in the 2 flavor system. To this end, calculations are performed for zero temperature under an instantaneous approximation while full $\mathbf{k}$-dependence is retained. That is, we allow strong coupling in the sense that momenta far from the Fermi surface also contribute. We will mention briefly the frequency dependence later.

We start from deriving the gap equation for $\Delta(k)$ representing the gap for color $\mathbf{3}^{*}$, isosinglet ${ }^{1} S_{0}$ pairs composed of two quarks which are time-reversal conjugate to each other with respect to space and spin,

$$
\begin{aligned}
& \Delta_{\mathbf{k} s f i, \mathbf{k}^{\prime} s^{\prime} f^{\prime} i^{\prime}}=(-1)^{\frac{1}{2}-s} \delta_{\mathbf{k},-\mathbf{k}^{\prime}} \delta_{s,-s^{\prime}} \epsilon_{f f^{\prime}} \hat{\epsilon}_{i i^{\prime}} \Delta(k), \\
& \epsilon_{f f^{\prime}}=\left(\begin{array}{cc}
0 & 1 \\
-1 & 0
\end{array}\right), \hat{\epsilon}_{i i^{\prime}}=\left(\begin{array}{ccc}
0 & 1 & -1 \\
-1 & 0 & 1 \\
1 & -1 & 0
\end{array}\right),
\end{aligned}
$$

where $s, f$ and $i$ denote spin, flavor and color, respectively. Note that time-reversal is represented by $T$ times complex conjugate, and $T=-i C \gamma^{5}$. We work in a Hamiltonian formalism since it is more convenient than the Gor'kov formalism [32] in the present study in which only the pair condensate is considered. The latter might be more suitable when the coupling between the pair condensate and the chiral condensate [17] is considered. Such a formalism has already been developed for the nuclear system [7.9] although the $N-\bar{N}$ condensate is negligibly small [9] due to a large nucleon mass. The resulting $12 \times 12$ Hamiltonian matrix,

$$
\begin{aligned}
& \left(\begin{array}{cc}
E_{k}-\mu & \Delta \\
-\Delta^{*} & -\left(E_{k}-\mu\right)
\end{array}\right), \\
& E_{k}=\sqrt{\mathbf{k}^{2}+M_{q}^{2}}, \mu=E_{k_{\mathrm{F}}},
\end{aligned}
$$

is easily block-diagonalized to two $6 \times 6$ matrices by inspection. Then they are fully diagonalized along the lines of the 1 flavor case studied in Ref. [14]. By expressing the pair condensate in terms of the Bogoliubov coefficients, we obtain 


$$
\begin{aligned}
& \Delta(p)=-\frac{1}{8 \pi^{2}} \int_{0}^{\infty} \bar{v}(p, k) \frac{\Delta(k)}{E^{\prime}(k)} k^{2} d k \\
& E^{\prime}(k)=\sqrt{\left(E_{k}-E_{k_{\mathrm{F}}}\right)^{2}+3 \Delta^{2}(k)} .
\end{aligned}
$$

As for the residual interaction, we adopt the one gluon exchange interaction with the leading order screening. Although the gluon propagator therein contains a gauge-dependent term in general, it vanishes due to the equation of motion of the external quark [27]. Then we proceed to a static (instantaneous) approximation 14,15, 18. At this step, the dynamic magnetic screening drops. By performing a spin average and an angle integration to project out the $S$-wave component, we obtain

$$
\begin{aligned}
& \bar{v}(p, k)=-\frac{\pi}{3} \alpha_{\mathrm{s}} \frac{1}{p k E_{p} E_{k}} \\
& \times\left(\left(2 E_{p} E_{k}+2 M_{q}^{2}+p^{2}+k^{2}+m_{\mathrm{E}}^{2}\right) \ln \left(\frac{(p+k)^{2}+m_{\mathrm{E}}^{2}}{(p-k)^{2}+m_{\mathrm{E}}^{2}}\right)\right. \\
& \left.+2\left(6 E_{p} E_{k}-6 M_{q}^{2}-p^{2}-k^{2}\right) \ln \left|\frac{p+k}{p-k}\right|\right) \\
& m_{\mathrm{E}}^{2}=\frac{4}{\pi} \alpha_{\mathrm{s}} \mu^{2} .
\end{aligned}
$$

The running coupling constant is taken from Ref. [33] as in Refs. [15, 18],

$$
\begin{aligned}
& \alpha_{\mathrm{s}}\left(\mathbf{q}^{2}\right)=\frac{4 \pi}{9} \frac{1}{\ln \left(\frac{q_{\mathrm{max}}^{2}+q_{\mathrm{c}}^{2}}{\Lambda_{\mathrm{QCD}}^{2}}\right)}, \\
& \mathbf{q}=\mathbf{p}-\mathbf{k}, q_{\max }=\max \{p, k\},
\end{aligned}
$$

with parameters $q_{\mathrm{c}}^{2}=1.5 \Lambda_{\mathrm{QCD}}^{2}, \Lambda_{\mathrm{QCD}}=0.4 \mathrm{GeV}$.

The frequency dependence of the gap, which is beyond the present formulation, has been shown recently to change the asymptotic behavior of the gap in the weak-coupling approaches [24,26,28]. There frequency dependence appears both in the gluon propagator that determines the interaction and in the other part of the integrand in the Eliashberg equation 24,26,23. Only the effects of the former can be estimated approximately here by modifying the magnetic part of Eq.(化) as

$$
\begin{aligned}
& -\frac{\pi}{3} \alpha_{\mathrm{s}} \frac{1}{p k E_{p} E_{k}} \\
& \times\left(6 E_{p} E_{k}-6 M_{q}^{2}-p^{2}-k^{2}-m_{\mathrm{M}}^{2}\right) \ln \left(\frac{(p+k)^{2}+m_{\mathrm{M}}^{2}}{(p-k)^{2}+m_{\mathrm{M}}^{2}}\right), \\
& m_{\mathrm{M}}^{2}=\left(\left|q^{0}\right| \alpha_{\mathrm{s}} \mu^{2}\right)^{2 / 3},
\end{aligned}
$$

where $m_{\mathrm{M}}^{2}$ is originating from the Landau damping [26,27] and the frequency $q^{0}$ is an independent parameter here due to the nature of the present formulation. Evidently this simply weakens the magnetic attraction and accordingly monotonically reduces the gap at the Fermi surface, $\Delta\left(q^{0}=10 \mathrm{MeV}\right) / \Delta\left(q^{0}=0\right)=0.70, \Delta\left(q^{0}=50 \mathrm{MeV}\right) / \Delta\left(q^{0}=0\right)=$ 0.53 , at $k_{\mathrm{F}}=2 \mathrm{fm}^{-1}$, for example, while the $q^{0}$-dependence of the coherence length (defined 
later) is rather weak. In the Eliashberg equation the gap is given as a function of $p^{0}$, where $q^{0}=p^{0}-k^{0}$ and the equation includes the integration with respect to $k^{0}$. Its $p^{0}$-dependence was numerically studied in Refs. [26,223, where the gap exhibits a maximum at intermediate densities whereas decreases monotonically at asymptotically high densities.

In the following, we concentrate on the static case and present numerical results in three steps. As for the quark mass, $M_{q}=10 \mathrm{MeV}$ is adopted according to Ref. [14]. First we discuss the gap at the Fermi surface $\Delta\left(k_{\mathrm{F}}\right)$ and the coherence length $\xi$ as functions of the Fermi momentum $k_{\mathrm{F}}$, which is related to the baryon density as $\rho=2 k_{\mathrm{F}}^{3} / 3 \pi^{2}$ in the present 2 flavor case. The coherence length is defined as [34],

$$
\xi=\left(\frac{\int_{0}^{\infty}\left|\frac{d \phi}{d k}\right|^{2} k^{2} d k}{\int_{0}^{\infty}|\phi|^{2} k^{2} d k}\right)^{1 / 2}
$$

in the strong coupling case, in terms of the pair wave function,

$$
\phi(k)=\frac{1}{2} \frac{\Delta(k)}{E^{\prime}(k)},
$$

which is identical to the pair condensate up to phase factors. In Fig.1 $1(\mathrm{a}), \Delta\left(k_{\mathrm{F}}\right)$ is graphed for $k_{\mathrm{F}}=1.5-3.25 \mathrm{fm}^{-1}$, which corresponds to $\rho / \rho_{0} \simeq 1.5-15$ if the normal density of symmetric $(N=Z)$ nuclear matter is defined as $\rho_{0}=2\left(k_{\mathrm{F}}\right)_{0}^{3} / 3 \pi^{2},\left(k_{\mathrm{F}}\right)_{0}=1.30 \mathrm{fm}^{-1}$ 35]. This shows very weak $k_{\mathrm{F}}$-dependence; the superconducting phase survives up to practically infinite density; for example, $\Delta\left(k_{\mathrm{F}}=60 \mathrm{fm}^{-1}\right) \simeq 50 \mathrm{MeV}$. We confirmed that the gap is predominantly brought about by the magnetic interaction; about $50 \%$ of the total gap remains when the electric interaction is cut artificially while only $5-10 \%$ remains when the magnetic part is cut. This magnetic dominance can also be deduced from the strong $k_{\mathrm{F}}$-dependence of $\bar{v}\left(k_{\mathrm{F}}, k\right)$ (Fig.2( (c) below) except at $k \simeq k_{\mathrm{F}}$ where the magnetic interaction gives very strong attraction irrespective of $k_{\mathrm{F}}$, in contrast to the weak $k_{\mathrm{F}}$-dependence of $\Delta\left(k_{\mathrm{F}}\right)$. The coherence length is shown in Fig.1 1 (b). This can be compared with the Pippard length in the weak coupling theory, $\xi_{0}=k_{\mathrm{F}} / \pi \Delta\left(k_{\mathrm{F}}\right) \mu \simeq 1 / \pi \Delta\left(k_{\mathrm{F}}\right)$ (see for example, Ref. [36]); the magnitudes of $\xi$ are approximated fairly well by $\xi_{0}$ whereas the $k_{\mathrm{F}}$-dependence is different. The average interparticle distance $d=\left(\frac{\pi^{2}}{2}\right)^{1 / 3} / k_{\mathrm{F}}$, derived from $\rho_{q}=3 \rho=1 / d^{3}$, is also shown in the figure. The magnitudes of $\xi$ and $d$ are very similar to each other as in the nucleon-nucleon case [10], whereas $\xi$ is $3-4$ orders of magnitude larger than $d$ in metals. This indicates strong coupling feature in the sense that quark Cooper pairs are compact and therefore bosonic. That is, fermion exchange is less when their mutual overlap is small. If $M_{q}$ is changed to $100 \mathrm{MeV}$ and $300 \mathrm{MeV}$, for example, $\Delta\left(k_{\mathrm{F}}\right)$ decreases to $90-98 \%$ and $54-81 \%$ of the original values, respectively, for $k_{\mathrm{F}}=1.5-3.25 \mathrm{fm}^{-1}$, whereas the changes in $\xi$ are negligibly small.

Another quantity which can be compared with $\xi$ is the London penetration depth $\lambda_{\mathrm{L}}$, which is defined by $\sqrt{\mu / 4 \pi e_{\mathrm{s}}^{2} \rho_{\mathrm{s}}}$ in the relativistic case, where $e_{\mathrm{s}}$ and $\rho_{\mathrm{s}}$ are the electric charge of the Cooper pair and the density of the superconducting component, respectively. This expression can be derived from the relativistic Ginzburg-Landau theory developed in Ref. [4] (see also Refs. [30,25]). In the present ud 2 flavor case, $\lambda_{\mathrm{L}} \simeq 20-8 \mathrm{fm}$ for $k_{\mathrm{F}}=1.5$ $-3.25 \mathrm{fm}^{-1}$ is obtained by adopting $e_{\mathrm{s}}=\frac{2}{3} e+\left(-\frac{1}{3}\right) e$ and $\rho_{\mathrm{s}}=\frac{2}{3} \rho_{q}$. Although Bailin and Love concluded that quark matter is a superconductor of the first kind, $\xi>\sqrt{2} \lambda_{\mathrm{L}}$, based 
on their estimate, $\Delta\left(k_{\mathrm{F}}\right) \sim 1 \mathrm{MeV}$ [4], the present quantitative study shows that quark matter is a superconductor of the second kind at these densities as pointed out in Ref. [30. At asymptotically high densities, however, it changes to a superconductor of the first kind since the penetration depth decreases rapidly as density increases. One should note that these discussion applies only to a part of magnetic field since the original photon and a gluon combine to a massless 'rotated' photon although both the electromagnetic U(1) and the color $\mathrm{SU}(3)$ break in color superconductors as recently discussed in Ref. [37.

Next, we turn to $k$-dependence at each $k_{\mathrm{F}}$. The compactness of the Cooper pairs mentioned above indicates spreading of $\Delta(k)$ (Fig.2(a)) and $\phi(k)$ (Fig.2(b)) in $k$-space. Figure 2(a) shows that $\Delta(k)$ spreads to larger $k$ at higher densities as expected, while the width of $\phi(k)$ in Fig.2(b), which corresponds to $1 / \xi$, is almost $k_{\mathrm{F}}$-independent as mentioned above since the quasiparticle energy $E^{\prime}(k)$ in the denominator in Eq.(7) grows as $k$ goes away from $k_{\mathrm{F}}$. The asymmetric shapes of $\phi(k)$ reflect the smallness of $\xi$. Its width is similar to that of $\bar{v}\left(k_{\mathrm{F}}, k\right)$ in Fig.2(c).

Finally, we Fourier-transform $\phi(k)$ to

$$
\phi(r)=\frac{1}{2 \pi^{2}} \int_{0}^{\infty} \phi(k) j_{0}(k r) k^{2} d k
$$

where $j_{0}(k r)$ is a spherical Bessel function, in order to look into the spatial structure of quark Cooper pairs more closely. This quantity has already been discussed for nucleon Cooper pairs in non-relativistic [39,40] and relativistic [10] studies. Although the coherence length $\xi$, corresponding to the root mean square distance with respect to this wave function, is almost $k_{\mathrm{F}}$-independent, the shape of $\phi(r)$ is strongly $k_{\mathrm{F}}$-dependent; large- $k$ components in high-density cases bring nodes in $r$-space. Conversely, $\phi(r)$ is diffuse at low densities. In other words, quark Cooper pairs become more bosonic at higher densities.

To summarize, we have studied numerically the spatial structure of quark Cooper pairs in the color $3^{*}$, isosinglet ${ }^{1} S_{0}$ channel by solving the gap equation in full momentum range. Although the long-range magnetic interaction is predominantly responsible for the quarkquark pairing, the gap function spreads also in momentum space. The resulting coherence length is almost density independent as well as the gap at the Fermi surface and is of magnitudes similar to the average interquark distance. The dependence of the pair wave function (condensate) on the relative momentum and on the distance between two quarks that form a Cooper pair has been presented. This indicates that quark Cooper pairs become more bosonic at higher densities although the coherence length is almost density independent.

The author thanks T. Hatsuda for informing him of R. Horie's master thesis in Ref. [18] and for the communication of Ref. [38]. 


\section{REFERENCES}

[1] M. A. Halasz, A. D. Jackson, R. E. Shrock, M. A. Stephanov and J. J. M. Verbaarschot, Phys. Rev. D 58, 096007 (1998).

[2] J. Berges and K. Rajagopal, Nucl. Phys. B538, 215 (1999).

[3] K. Langfeld and M. Rho, hep-ph/9811227.

[4] D. Bailin and A. Love, Nucl. Phys. B190, 175; 751 (1981); ibid. B205, 119 (1982); Phys. Rep. 107, 325 (1984).

[5] B. C. Barrois, Nucl. Phys. B129, 390 (1977).

[6] H. Kucharek and P. Ring, Z. Phys. A339, 23 (1991).

[7] F. B. Guimarães, B. V. Carlson and T. Frederico, Phys. Rev. C 54, 2385 (1996).

[8] F. Matera, G. Fabbri and A. Dellafiore, Phys. Rev. C 56, 228 (1997).

[9] M. Matsuzaki, Phys. Rev. C 58, 3407 (1998).

[10] T. Tanigawa and M. Matsuzaki, Prog. Theor. Phys. 102, 897 (1999); M. Matsuzaki and T. Tanigawa, nucl-th/9911042.

[11] L. A. Kondratyuk, M. M. Giannini and M. I. Krivoruchenko, Phys. Lett. B269, 139 (1991); L. A. Kondratyuk and M. I. Krivoruchenko, Z. Phys. A344, 99 (1992).

[12] J. C. R. Bloch, C. D. Roberts and S. M. Schmidt, Phys. Rev. D 60, 065208 (1999).

[13] S. Hands, J. B. Kogut, M. -P. Lombardo and S. E. Morrison, hep-lat/9902034; J. B. Kogut, M. A. Stephanov and D. Toublan, Phys. Lett. B464, 183 (1999).

[14] M. Iwasaki and T. Iwado, Phys. Lett. B350, 163 (1995); Prog. Theor. Phys. 94, 1073 (1995).

[15] M. Iwasaki, Prog. Theor. Phys. 101, 91 (1999).

[16] R. Rapp, T. Schäfer, E. Shuryak and M. Velkovsky, Phys. Rev. Lett. 81, 53 (1998).

[17] M. Alford, K. Rajagopal and F. Wilczek, Phys. Lett. B422, 247 (1998).

[18] R. Horie, master thesis (Kyoto University, 1999); T. Hatsuda, Kyoto University preprint KUNS-1573 (1999).

[19] M. Alford, K. Rajagopal and F. Wilczek, Nucl. Phys. B537, 443 (1999).

[20] T. Schäfer and F. Wilczek, Phys. Rev. Lett. 82, 3956 (1999).

[21] D. K. Hong, hep-ph/9905523; D. K. Hong, M. Rho and I. Zahed, Phys. Lett. B468, 261 (1999).

[22] R. D. Pisarski and D. H. Rischke, nucl-th/9907094.

[23] N. Evans, J. Hormuzdiar, S. D. H. Hsu and M. Schwetz, hep-ph/9910313.

[24] D. T. Son, Phys. Rev. D 59, 094019 (1999).

[25] D. K. Hong, hep-ph/9812510; D. K. Hong, V. A. Miransky, I. A. Shovkovy and L. C. R. Wijewardhana, hep-ph/9906478.

[26] T. Schäfer and F. Wilczek, Phys. Rev. D 60, 114033 (1999).

[27] S. D. H. Hsu and M. Schwetz, hep-ph/9908310.

[28] R. D. Pisarski and D. H. Rischke, nucl-th/9907041.

[29] W. E. Brown, J. T. Liu and H. -c. Ren, hep-ph/9908248.

[30] D. Blaschke, D. M. Sedrakian and K. M. Shahabasyan, astro-ph/9904395.

[31] D. Blaschke, T. Klähn and D. N. Voskresensky, astro-ph/9908334.

[32] L. P. Gor'kov, Sov. Phys. JETP 7, 505 (1958).

[33] K. Higashijima, Prog. Theor. Phys. Suppl. 104, 1 (1991).

[34] F. V. De Blasio, M. Hjorth-Jensen, Ø. Elgarøy, L. Engvik, G. Lazzari, M. Baldo and H. -J. Schulze, Phys. Rev. C 56, 2332 (1997). 
[35] B. D. Serot and J. D. Walecka, Int. J. Mod. Phys. E6, 515 (1997).

[36] P. G. de Gennes, Superconductivity of Metals and Alloys, (Perseus Books Publishing, Massachusetts, 1989).

[37] M. Alford, J. Berges and K. Rajagopal, hep-ph/9910254.

[38] T. Hatsuda, private communication (1999).

[39] M. Baldo, J. Cugnon, A. Lejeune and U. Lombardo, Nucl. Phys. A515, 409 (1990).

[40] Ø. Elgarøy, L. Engvik, M. Hjorth-Jensen and E. Osnes, Nucl. Phys. A604, 466 (1996). 


\section{FIGURES}

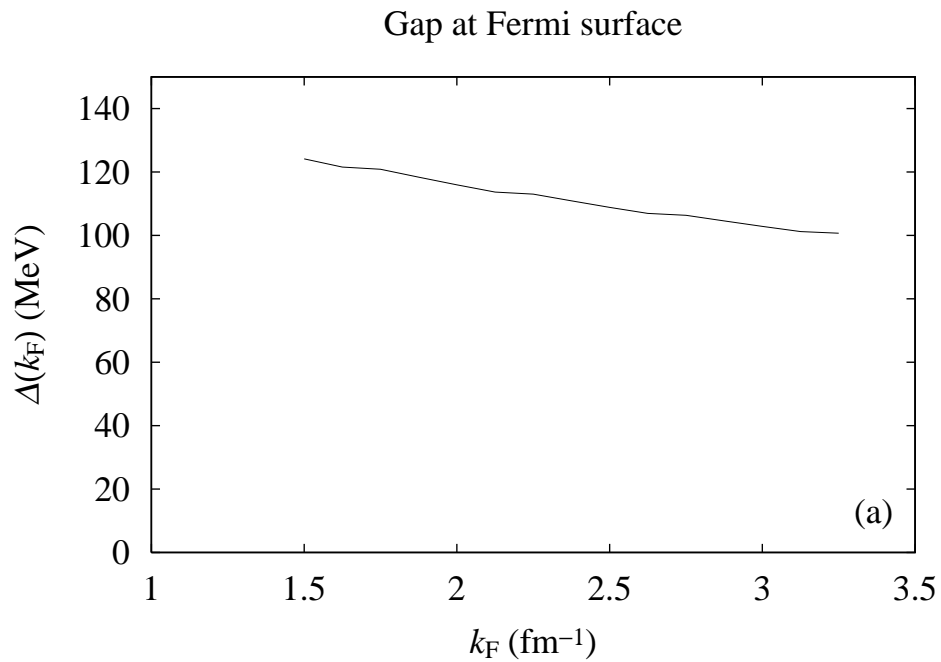

Coherence length and Interparticle distance

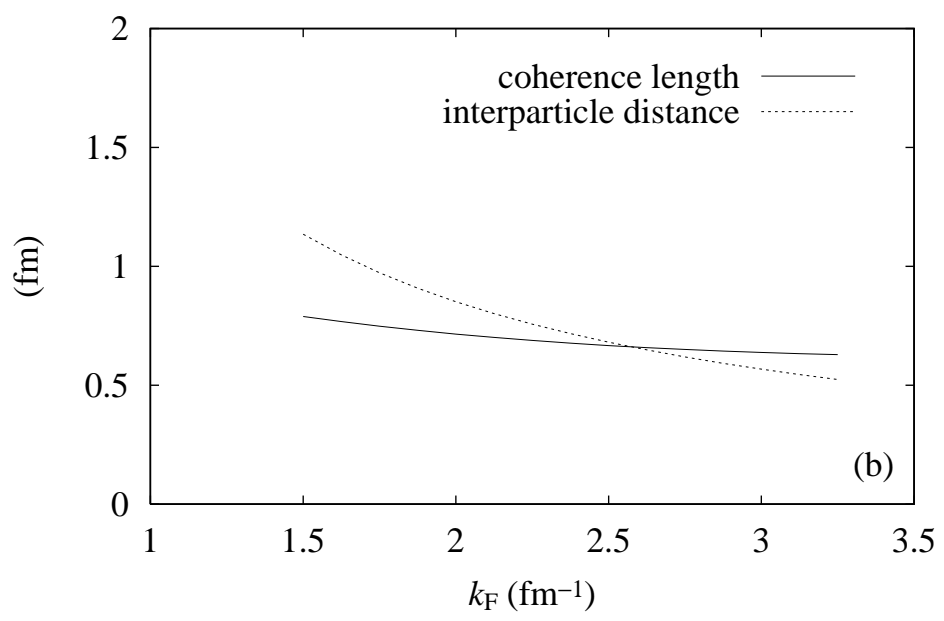

FIG. 1. (a) Pairing gap at the Fermi surface, and (b) coherence length and average interquark distance, as functions of the Fermi momentum $k_{\mathrm{F}}$. 

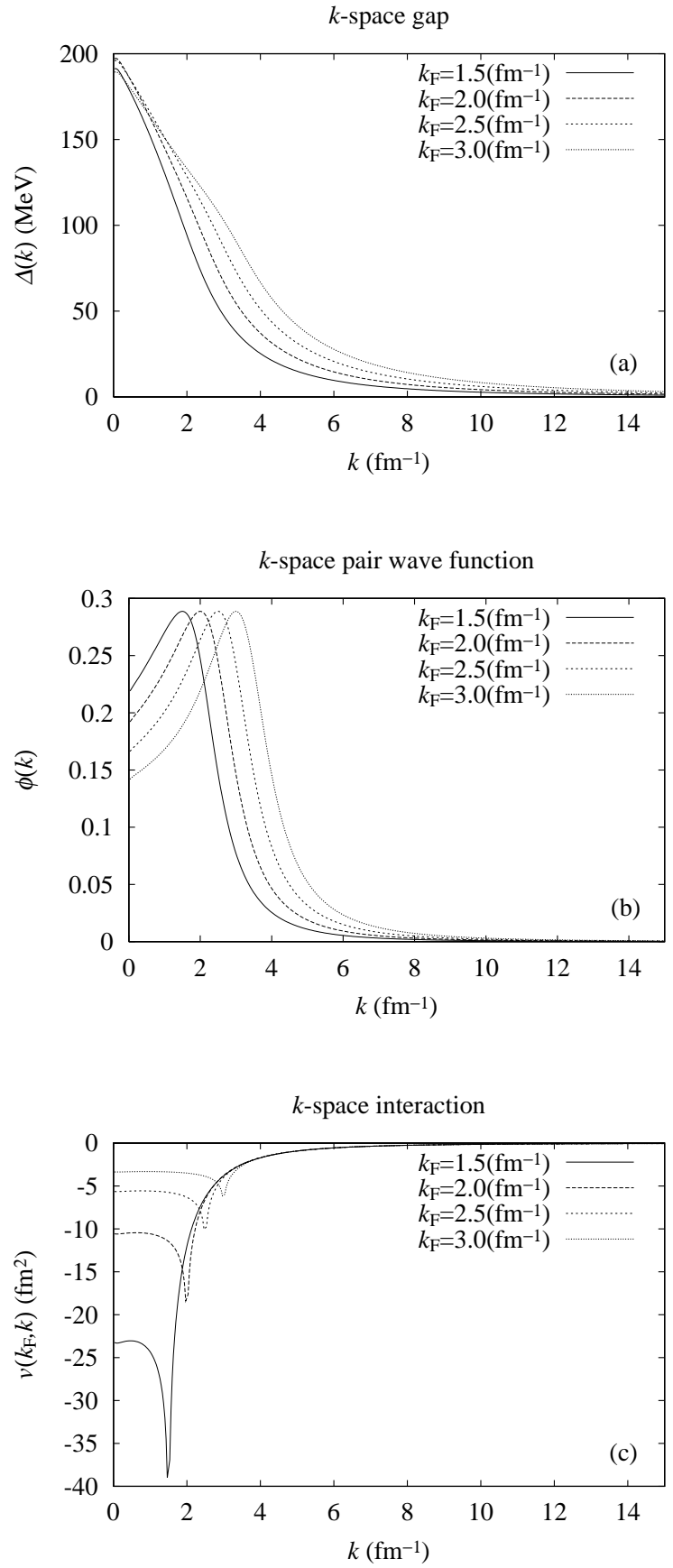

FIG. 2. (a) Gap function, (b) pair wave function, and (c) matrix element $\bar{v}\left(k_{\mathrm{F}}, k\right)$, as functions of the momentum $k$, calculated at $k_{\mathrm{F}}=1.5-3.0 \mathrm{fm}^{-1}$. Note that, in (c), $k=k_{\mathrm{F}}$ is excluded since at this point the diverging magnetic interaction does not contribute to the gap equation [38]. 


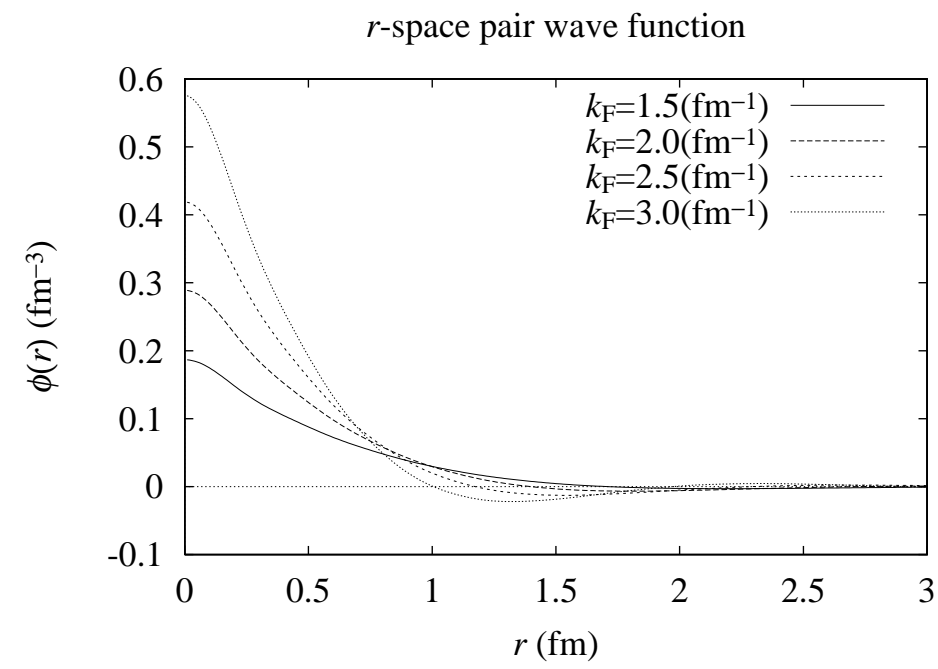

FIG. 3. Pair wave function as a function of the distance $r$, calculated at $k_{\mathrm{F}}=1.5-3.0 \mathrm{fm}^{-1}$. 